\title{
Bioanalysis
}

\section{Robust Ki67 detection in human blood by flow cytometry for clinical studies}

\begin{abstract}
Aim: Ki67 is a prognostic and/or predictive biomarker in patients with malignancies. Flow cytometry is a powerful technology for single-cell multiparameter analysis. Results: We developed and validated a multicolor quantitative flow cytometry assay for detection of intracellular Ki67 expression in various immune cell subsets from human blood. The assay was optimized and showed excellent precisions. Assessment of the sample stability indicated that percentage changes from the fresh sample for the reportable results of interest were within $20 \%$, up to $72 \mathrm{~h}$ after blood collection in the Cyto-Chex ${ }^{\circledast}$ BCT tube. Conclusion: The validated assay is sufficiently robust to analyze clinical samples. Easy access to peripheral blood enables continuous monitoring of Ki67 expression in blood as a biomarker, for example, for immunotherapy studies.
\end{abstract}

First draft submitted: 15 July 2016; Accepted for publication: 6 October 2016; Published online: 3 November 2016

Keywords: assay validation $\bullet$ biomarker $\bullet$ flow cytometry assay $\bullet$ immunotherapy $\bullet$ Ki67

Cancer immunotherapy has made a significant impact in the field of cancer treatment, from reshaping therapeutic concepts to raising the standard for care $[1,2]$. The recent approval of immune checkpoint blockade antibodies, which target cytotoxic T-lymphocyte-associated antigen- 4 and programmed cell death protein-1 in melanoma, non-small-cell lung cancer and kidney cancer, has supported this trend $[3,4]$. In spite of this success, limitations still exist. Some cancer patients do not respond to such therapies; those who do respond often experience toxicities. To advance our understanding in this area, there is a growing need to identify predictive and prognostic biomarkers [5,6]. In this way, we can stratify patients, track tumor-specific immune responses and study the immune system's complex interactions with cancer.

Ki67, a cell proliferation marker, has been used as a prognostic and/or predictive biomarker in patients with breast cancer and other tumors. Immunohistochemistry is commonly used to assess the expression of Ki67 in tumor tissues, but has limitations around assay standardization and sample accessibility [7-10]. Therefore, there is an unmet need to develop a quantitative and standardized assay that can measure Ki67 expression from an easily accessible specimen.

Flow cytometry has been a powerful technology, due to its unique ability to analyze large numbers of single cells with multiple parameters that can be measured quantitatively at the same time. In addition, the ideal sample for flow cytometry studies is human blood, which is more accessible than tumor tissues. Previously, this technology had been limited to preclinical studies, but recent efforts to recommend best practices and standardization in flow cytometry have made this technology feasible in clinical trials as well [11,12].

There have been several reports of Ki67 detection in human blood using flow cytometry [13-17]. To support global clinical trials, a robust Ki67 flow cytometry assay
Yongliang Sun', Katherine Yang ${ }^{1}$, Terry Bridal ${ }^{2}$ \& Anka G Ehrhardt ${ }^{*}, 1$

${ }^{1}$ Clinical Cytometry, Clinical Translational Technologies \& Operations, Bristol-Myers Squibb Co., 311 Pennington-Rocky Hill Road, Pennington, NJ 08534, USA

${ }^{2}$ Aerotek Scientific Resources, 371 Hoes Lane, Piscataway, NJ 08854, USA

*Author for correspondence:

Tel.: +1 6098184822

Anka.Ehrhardt@bms.com

\section{FUTURE SCIENCE prator}


is needed. Therefore, we have developed and validated a multicolor flow cytometry assay to measure intracellular Ki67 expression in various immune cell subsets from human blood. Since peripheral blood is easily accessible, changes of Ki67 expression can be measured at multiple points before and after a therapeutic treatment that targets the immune system [17]. Given the dynamic, complex relationship between the tumor and immune system, our developed assay will be very useful for evaluating a patient's real-time response to immunotherapy, as the expression of $\mathrm{Ki} 67$ can be continuously measured using human blood.

\section{Experimental}

Collection of blood samples

Human blood from healthy adults was collected with informed consent under the Bristol-Myers Squibb Institutional Review Board approved protocol using Cyto-Chex ${ }^{\circledR}$ BCT (Streck, Inc., NE, USA) and sodium heparin collection tubes (Becton-Dickinson VACUTAINER Systems, NJ, USA). Both types of collection tubes are designed to collect blood sample by standard venipuncture. Blood samples were stored at ambient temperature until analysis.

\section{Reagents \& monoclonal antibodies}

The monoclonal antibodies used in the study are listed in Table 1. In addition, anti-Ki67 (clone Ki67)PerCP-Cy5.5 and anti-Ki67 (clone 20Raj1)-PerCPeFluor710 were purchased from BioLegend (Cat\# 350520, CA, USA) and eBioscience (Cat\# 46-5699, CA, USA), respectively. BD Horizon ${ }^{\mathrm{TM}}$ Brilliant Stain Buffer (BD Biosciences, CA, USA, cat\#563794) was included to prepare the antibody cocktail. We tested different permeabilization buffers, which included BD Pharmingen ${ }^{\mathrm{TM}}$ Transcription Factor (BD Biosciences, cat\# 562574), BD Cytofix/Cytoperm ${ }^{\mathrm{TM}}$ fixation and permeabilization solution (BD Biosciences, cat\# 554714), BioLegend True-Nuclear ${ }^{\mathrm{TM}}$
Transcription Factor Buffer (BioLegend, cat\# 424401) and eBioscience Foxp3/Transcription Factor Staining Buffer (eBioscience, cat\# 00-5523). Lysis of red blood cells was performed using BD FACS ${ }^{\mathrm{TM}}$ lysing solution (BD Biosciences, cat\# 349202) according to the manufacturer's instructions.

\section{Multiparameter flow cytometric staining of peripheral blood samples}

Two hundred microliters of whole blood was added to a $12 \times 75 \mathrm{~mm}$ BD FACS test tube, and first stained with antibodies against the cell-surface markers as described in Table 1, for $30 \mathrm{~min}$ at room temperature in the dark. To lyse RBCs, $4 \mathrm{ml}$ of $1 \times$ BD FACS lysing solution was added, mixed well and incubated for $15 \mathrm{~min}$ at room temperature in the dark. After centrifugation at $500 \times g$ at $4^{\circ} \mathrm{C}$, the cells were washed once with $4 \mathrm{ml}$ of stain buffer (BD Biosciences, cat\#554657). Before permeabilization, the cell pellet was thoroughly resuspended. The permeabilization procedure was performed according to each manufacturer's protocol. For BD Cytofix/Cytoperm ${ }^{\mathrm{TM}}$ fixation/permeabilization buffer that was ultimately chosen for this assay, $250 \mu \mathrm{l}$ of BD Cytofix/ Cytoperm solution was added per tube and vortexed well. After incubating for $20 \mathrm{~min}$ at $4^{\circ} \mathrm{C}$, the cells were washed twice in $1 \times \mathrm{BD}$ Perm/Wash buffer (BD Biosciences, CA, USA), $3 \mathrm{ml}$ per tube. The permeabilized cells were thoroughly resuspended in $100 \mu \mathrm{l}$ of $1 \times$ BD Perm/Wash buffer before the Ki67 intracellular staining. A titrated anti-Ki67 fluorochromeconjugated antibody was added and incubated at $4^{\circ} \mathrm{C}$ for $60 \mathrm{~min}$ in the dark. A test tube without addition of the anti-Ki67 antibody was used as Fluorescence Minus One control. The stained cells were washed twice with $3 \mathrm{ml}$ of $1 \times$ BD Perm/Wash buffer, and then resuspended in $300 \mu \mathrm{l}$ of the stain buffer prior to flow cytometric analysis using a BD FACSCanto ${ }^{\mathrm{TM}}$ System (BD Biosciences).

\section{Table 1. Panel information.}

\begin{tabular}{|lllllll|}
\hline Marker & Clone & Fluorochrome & $\mu$ ltest & Staining & Catalog \# & Vendor \\
CD3 & UCHT1 & BV510 & 2.5 & Surface & 300448 & BioLegend \\
\hline CD8 & SK1 & APC-H7 & 2.5 & Surface & 560179 & BD Biosciences \\
\hline CD4 & RPA-T4 & AF700 & 1.0 & Surface & 300526 & BioLegend \\
\hline CD45RA & H1100 & AF488 & 2.5 & Surface & 304114 & BioLegend \\
\hline CD56 & HCD56 & AF647 & 2.5 & Surface & 318314 & BioLegend \\
\hline CCR7 & GO4387 & BV421 & 2.5 & Surface & 353208 & BioLegend \\
\hline CD127 & A019D5 & PE-Cy7 & 2.5 & Surface & 351320 & BioLegend \\
\hline CD25 & 4E3 & PE & 2.5 & Surface & $12-0257-42$ & eBioscience \\
\hline Ki67 & B56 & PerCP-Cy5.5 & 5.0 & Intracellular & 561284 & BD Biosciences \\
\hline
\end{tabular}


Flow cytometry data acquisition \& analysis

A BD FACSCanto flow cytometer (4-3-3 configuration) was used to acquire data from stained samples. The instrument was calibrated daily with Becton Dickinson's Cytometer Setup and Tracking System beads using the Cytometer Setup and Tracking software. OneComp eBeads (eBioscience, cat\# 01-1111) were stained with individual fluorochrome-conjugated antibodies for use as single-color compensation controls. BD FACSDiva $^{\mathrm{TM}}$ software V8.01 (BD Biosciences) was used for data acquisition. For each sample, 45,000 events were collected using the $\mathrm{CD}_{4}{ }^{+}$cell gate as a stopping gate. Data analysis was performed using either Flowjo software V10.0.7 (Tree Star, Inc., OR, USA) or BD FACSDiva software V8.01.

\section{Validation}

A fit-for-purpose method validation strategy was applied for this assay $[11,18,19]$. To assess sample stability, aliquots were processed under aseptic conditions for analysis from each Cyto-Chex ${ }^{\circledR}$ BCT at 2, 24, 48, 72 and 96 $\mathrm{h}$ postcollection. Blood samples were processed within $2 \mathrm{~h}$ postcollection to establish a baseline measurement for stability determination. The cut-off time point was determined based on $<20 \%$ differences between the sample value at a given time point and the baseline sample value. Intra-assay precision was determined by assaying samples in triplicates, and the percentage $\mathrm{CV}$ was calculated within the triplicates. Interanalyst variability was assessed between two trained individuals by processing the same samples in parallel on the same day. The percentage differences between analysts were calculated. Interassay precision was assessed using commercially available flow cytometry control material lyophilized Veri-Cells ${ }^{\mathrm{TM}}$ PBMC (BioLegend, cat\#425002) assayed in triplicate in five independent analytical runs performed on five separate days. For each run, the mean was calculated for each reportable result. Then, the grand mean percentage $\mathrm{CV}$ was calculated from the five runs. To evaluate prepared sample stability, samples were prepared in triplicate, combined and divided into three aliquots. The first aliquot was acquired upon processing. The remaining two aliquots were held at $4^{\circ} \mathrm{C}$ for 4 and $24 \mathrm{~h}$ before acquisition. The same instrument was used for each acquisition that was completed within $30 \mathrm{~min}$. Percentage changes calculated from the first acquisition were generated for a stability assessment.

\section{Statistical analysis}

Graphpad Prism 5.0 (Graphpad Software, CA, USA) one-way analysis of variance (ANOVA) was used to compare frequencies of $\mathrm{Ki}^{+} 7^{+}$cells among various immune cell subsets. A p-value $<0.05$ was considered statistically significant.

\section{Results \& discussion}

Development of a flow cytometry assay to measure intracellular Ki67 expression in immune cell subsets from human blood

A nine-color flow cytometric panel was developed as shown in Table 1 using BD FACSCanto 10-configuration platform. We designed the panel based on antigen density, co-expression of the markers and literature [20]. Different assay conditions were tested to optimize the assay performance. Titration of the antibodies, clone selection for antibodies against the surface markers and fluorochrome combination were examined in multiple sets of experiments (data not shown). In addition, three commercially available anti-Ki67 antibody clones were compared as described below. Since it was an intracellular flow cytometry assay, permeabilization buffers from various vendors were compared and described below. Ultimately, we chose the assay conditions that provided the best performance in staining quality and separation of subpopulations of interest. Figure 1A illustrates the gating strategy for the panel. First, lymphocytes were gated based on forward-scatter area versus side-scatter area. After cell doublets were excluded using forward-scatter area versus forwardscatter height, $\mathrm{CD}^{+}$cells were gated, and then divided into $\mathrm{CD}^{+} \mathrm{CD}^{+}$and $\mathrm{CD} 3^{+} \mathrm{CD} 8^{+}$cells. For either $\mathrm{CD}^{+}{ }^{+} \mathrm{CD} 4^{+}$or $\mathrm{CD} 3{ }^{+} \mathrm{CD} 8{ }^{+}$cells, CCR7 and CD $45 \mathrm{RA}$ were used to further divide them into four subsets: naive $\left(\mathrm{CD} 45 \mathrm{RA}^{+} \mathrm{CCR} 7^{+}\right)$, central memory $(\mathrm{CD} 45 \mathrm{RA}$ CCR7 $7^{+}$), effector memory (CD45RA-CCR7-) and terminally differentiated effector memory CD45RA ${ }^{+}$ (CD45RA+CCR7 $)$. Treg were gated as $\mathrm{CD} 25^{\text {high }} \mathrm{CD}$ $127^{\text {low }}$ from $\mathrm{CD}^{+}$cells. Natural killer $\mathrm{T}$ cells were defined as $\mathrm{CD}^{+} \mathrm{CD} 56^{+}$. NK cells were defined as $\mathrm{CD}^{-} \mathrm{CD} 56^{+}$or $\mathrm{CD} 3 \mathrm{CD}^{-} 6^{\text {bright }}$ [20]. The frequency of $\mathrm{Ki} 67^{+}$cells was determined in each subset. Figure 1B shows the cumulative data of frequencies of $\mathrm{Ki} 67^{+}$cells in various immune cell subsets in blood from 14 healthy subjects. The percentage of $\mathrm{Ki} 67^{+}$cells varies, depending on the subset, indicating that each subset proliferates at different rates in vivo [13-15,17].

\section{Comparison of permeabilization buffers}

Intracellular multicolor flow cytometry is technically challenging due to the fact that the permeabilization should be sufficient to allow exposure of intracytoplasmic/intranuclear proteins to the detection antibody, and also be gentle enough to preserve staining of the surface markers. Since Ki67 is a nuclear protein [7], we compared the BD Pharmingen ${ }^{\mathrm{TM}}$ Transcription Factor to BD Cytofix/Cytoperm fixation and permeabilization solution, and found that the staining and separation of the surface markers and intracellular Ki67 staining were comparable (data not shown). Then we compared BD 


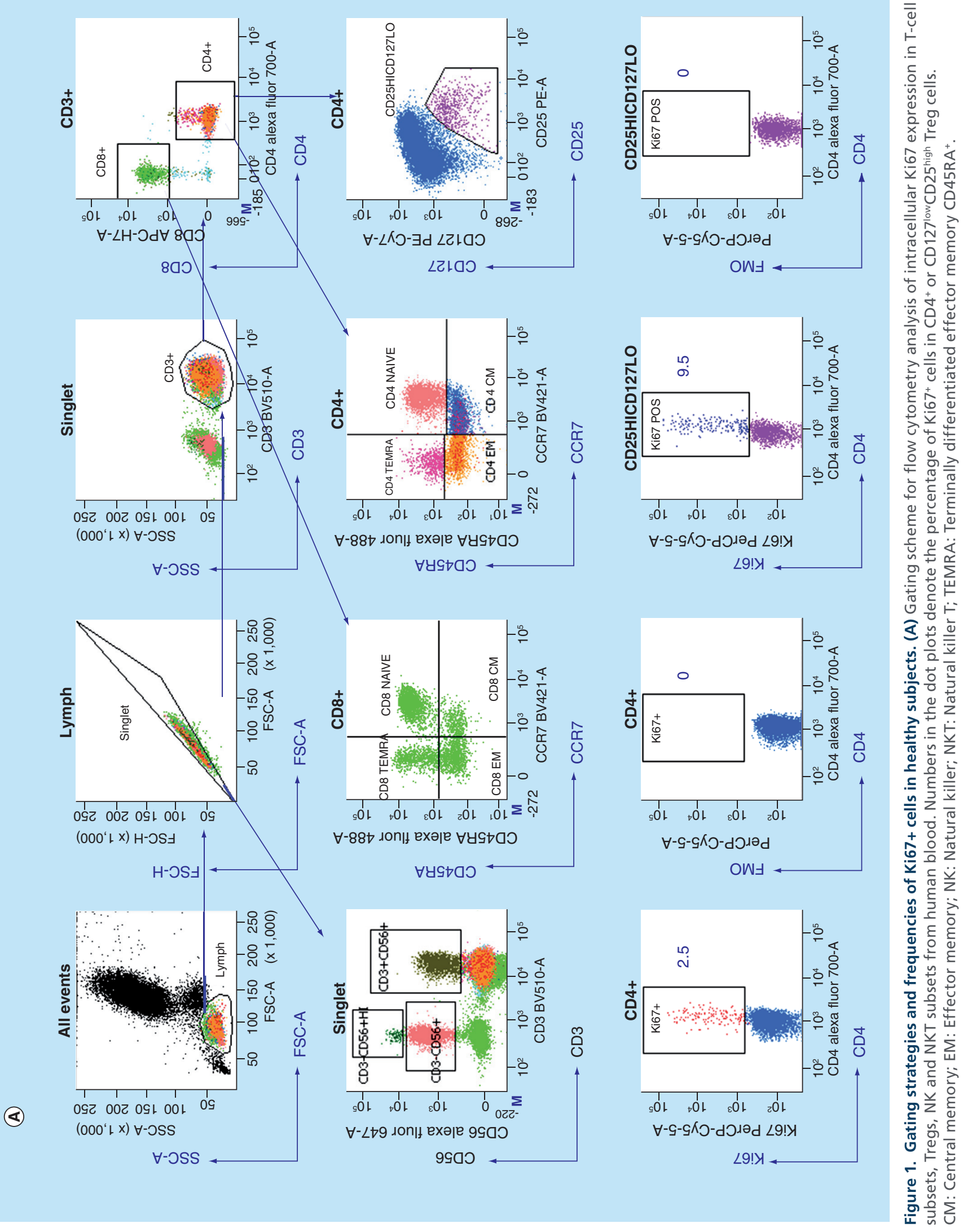




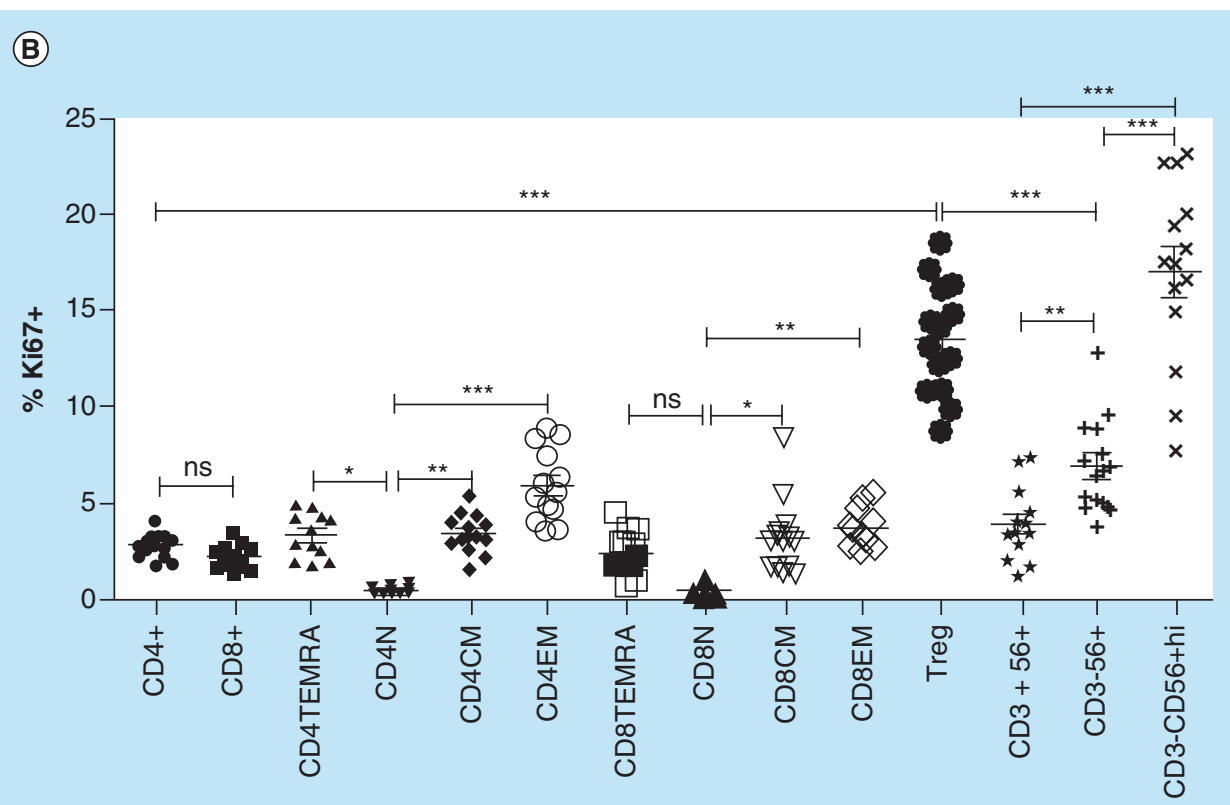

Figure 1. Gating strategies and frequencies of Ki67+ cells in healthy subjects (cont.). (B) Cumulative data showing frequencies of $\mathrm{Ki} 67^{+}$cells in each subset from 14 healthy subjects. Each symbol represents a different individual, and the mean frequency is shown as a horizontal line. Significance was determined by one-way ANOVA. * $p<0.05$, $* * p<0.01, * * * p<0.001$.

CM: Central memory; EM: Effector memory; N: Naive; ns: Not significant; TEMRA: Terminally differentiated effector memory CD45RA ${ }^{+}$.

Cytofix/Cytoperm fixation and permeabilization solution, BioLegend True-Nuclear ${ }^{\mathrm{TM}}$ Transcription Factor Buffer and eBioscience Foxp3/Transcription Factor Staining Buffer to look at the staining performance. Figure 2A shows a representative sample for head-to-head comparison using these three buffers. The frequency of $\mathrm{CD} 25^{\text {high }} \mathrm{CD} 127^{\text {low }}$ Tregs from $\mathrm{CD}^{+}$cells was similar between the $\mathrm{BD}$ and eBioscience buffers, and slightly lower in the BioLegend's buffer. The BD buffer provided better separation of surface marker CCR7 and CD45RA than the other two buffers. The Ki67 staining pattern and frequencies of $\mathrm{Ki}^{+} 7^{+}$in Treg cells, and other subsets (data not shown) were very similar for all the permeabilization buffers tested. Staining of all other surface markers was decent for all the tested permeabilization buffers (data not shown). Based on the above results, we chose BD Cytofix/Cytoperm buffer as it had the least effect on staining of the surface markers in the panel, and provided a consistent intracellular Ki67 staining. The minimum effect of permeabilization on the staining of surface markers was confirmed by comparing the intracellular staining to the surface staining only using aliquots of the same samples. The stained samples with or without permeabilization treatment were acquired using the same instrument settings. The differences in frequencies of subpopulations of interest between the two groups, as shown in Figure 2B were within $20 \%$. The difference in median fluorescence intensity of CD25 on Tregs was $6.02 \%$. As expected, no positive
Ki67 staining was observed in the stained samples without permeabilization. Selection of the permeabilization buffer with the least impact on staining of surface markers allows cross-panel data analysis for panels with or without intracellular markers. When multiple surface and intracellular panels are applied to a clinical study, it is important that shared markers across the panels are comparable in staining patterns and intensities.

\section{Ki67 clone matters}

We evaluated three commercially available anti-Ki67 antibody clones to look at the differences in $\mathrm{Ki67}$ staining pattern and frequency of $\mathrm{Ki}^{+} 7^{+}$cells. Clone B56 (BD Biosciences), Ki67 (BioLegend) and 20Raj1 (eBioscience) were compared. Aliquots of the same samples were assayed in parallel using the same instrument settings. As shown in Figure 3, the B56 and 20Raj1 clones provided almost identical Ki67 staining pattern, and similar frequencies of $\mathrm{Ki}^{+} 7^{+}$in both $\mathrm{CD} 4^{+}$ (Figure 3A \& C) and Treg cells (Figure 3B \& D). Whereas the frequency of $\mathrm{Ki}^{+} 7^{+}$cells using the Ki67 clone was approximately $50 \%$ in either $\mathrm{CD}^{+}$or Treg cells, as compared with the other two clones. Similar results were observed in other cell subsets (data not shown). Furthermore, we back gated $\mathrm{Ki}^{+} 7^{+}$cells to compare the distribution of these cells in the $\mathrm{CD}^{+}$cell subsets as defined by CCR7 and CD45RA. As shown in Figure $3 \mathrm{E}$, the distribution pattern of $\mathrm{Ki}^{+} 7^{+}$cells was similar regardless of clones. Most $\mathrm{Ki}^{+} 7^{+}$cells (blue 


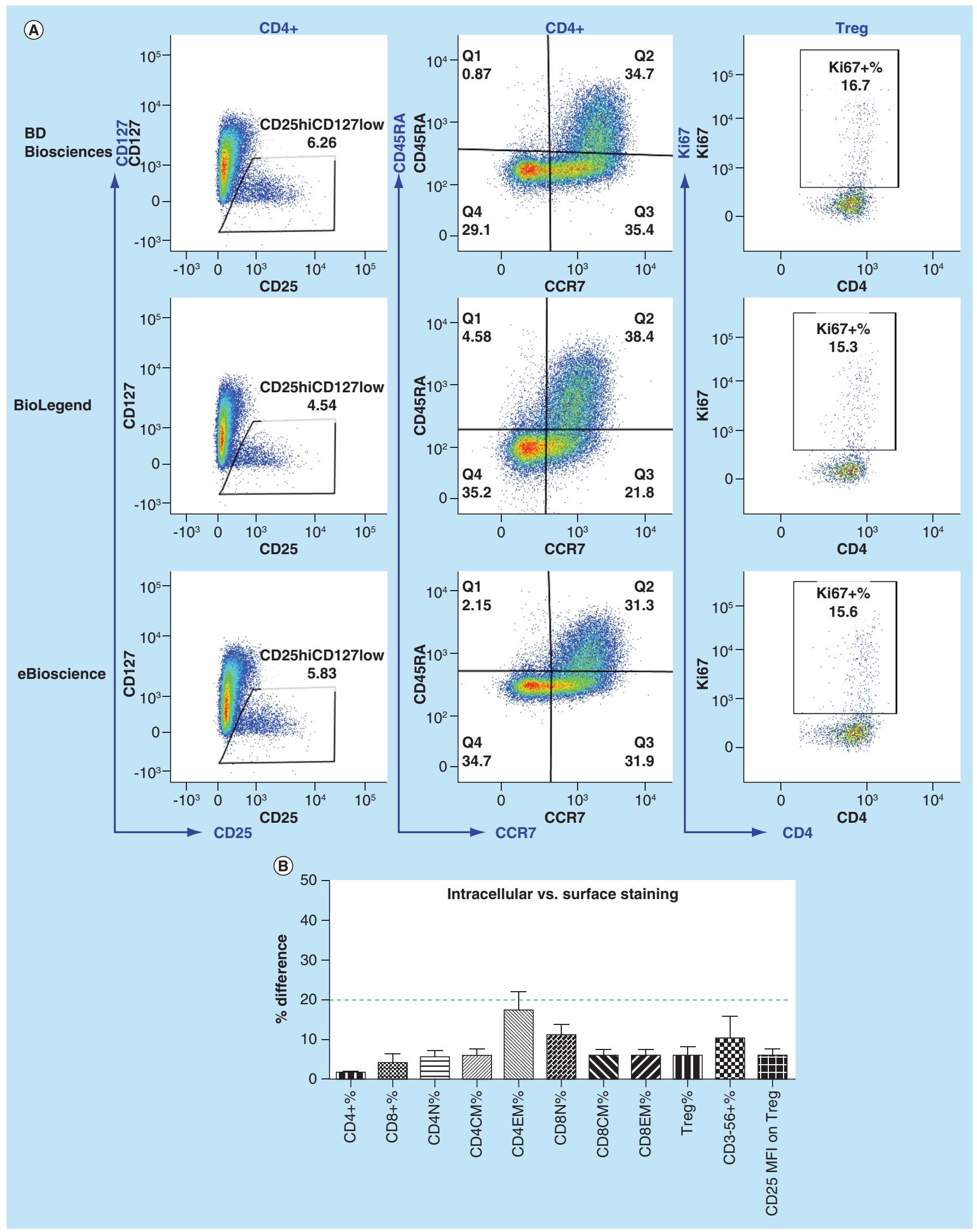

Figure 2. Effect of permeabilization on staining of surface markers. (A) Comparison of permeabilization buffers. Dot plots from a representative blood sample are shown. CD4+ Tregs were defined as CD25 high CD127low (left column); CD4+ subsets (N, CM, EM and TEMRA) were gated by CD45RA versus CCR7 (middle column) and Ki67+ Tregs were gated based on the FMO control (right column). Four healthy donor blood specimens were used for each experiment that was repeated three-times. (B) Comparison between the intracellular staining and the surface only staining. Four healthy donor blood specimens were processed in parallel with or without permeabilization. The average percentage differences between them were calculated. The green dot line indicates the $20 \%$ cut-off. The experiment was repeated twice.

CM: Central memory; EM: Effector memory; FMO: Fluorescence Minus One; N: Naive; TEMRA: Terminally differentiated effector memory CD45RA+. 
(A)

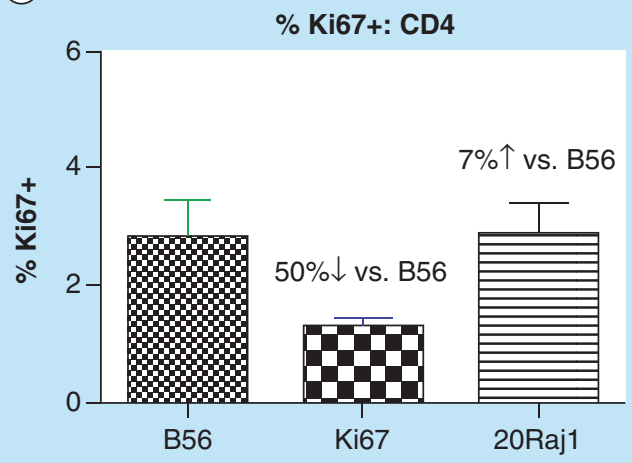

(B)

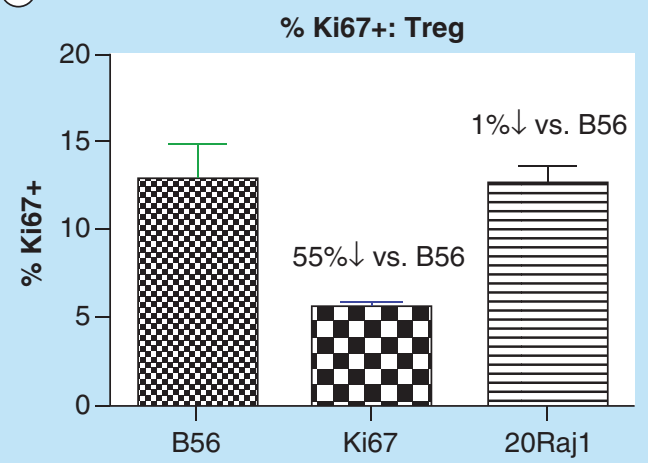

(C)

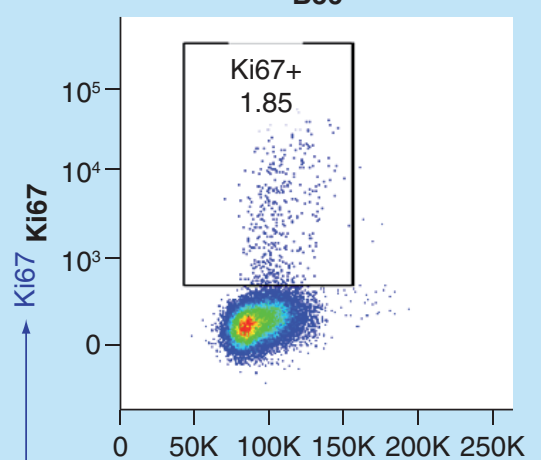

FSC-A

(D)

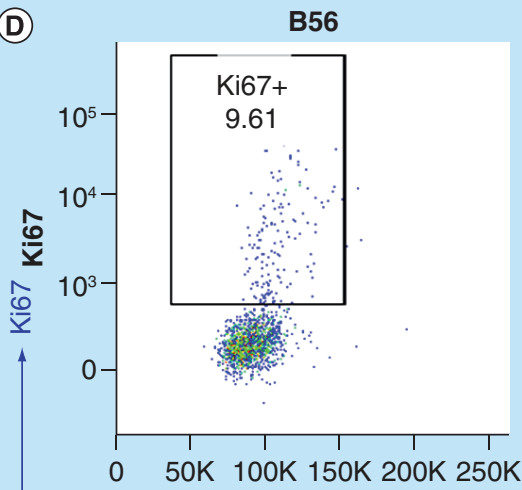

FSC-A

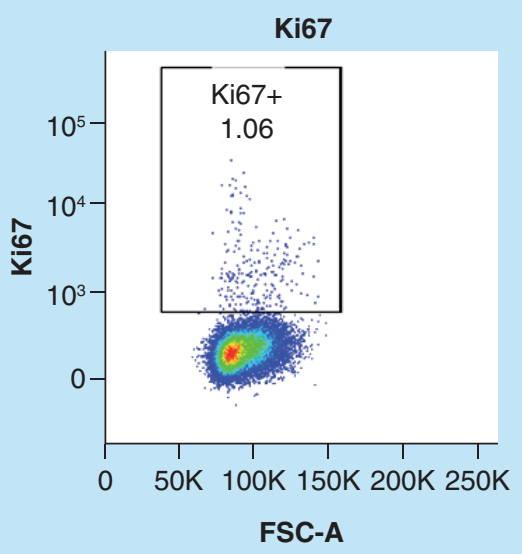

Ki67

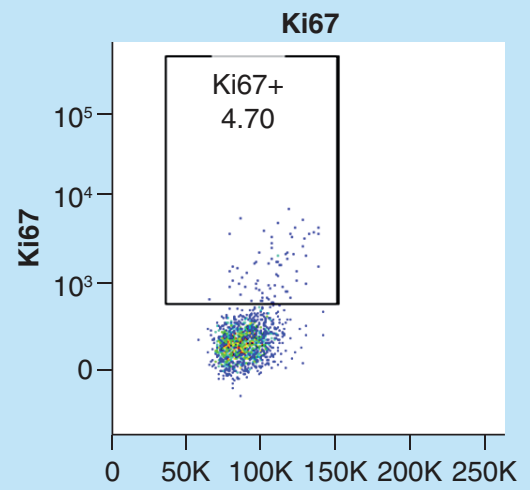

FSC-A

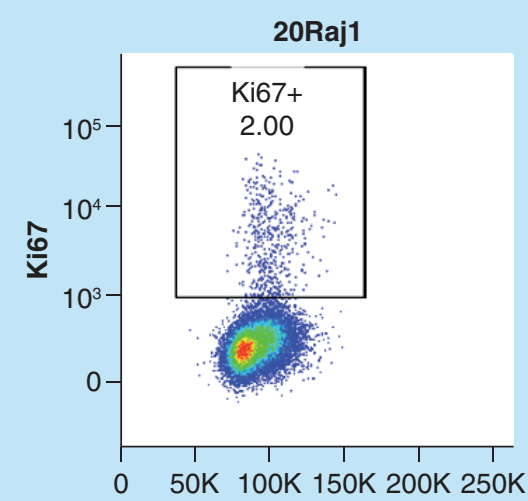

FSC-A

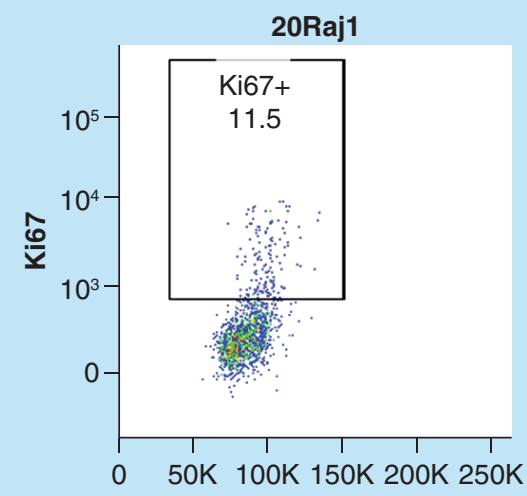

FSC-A
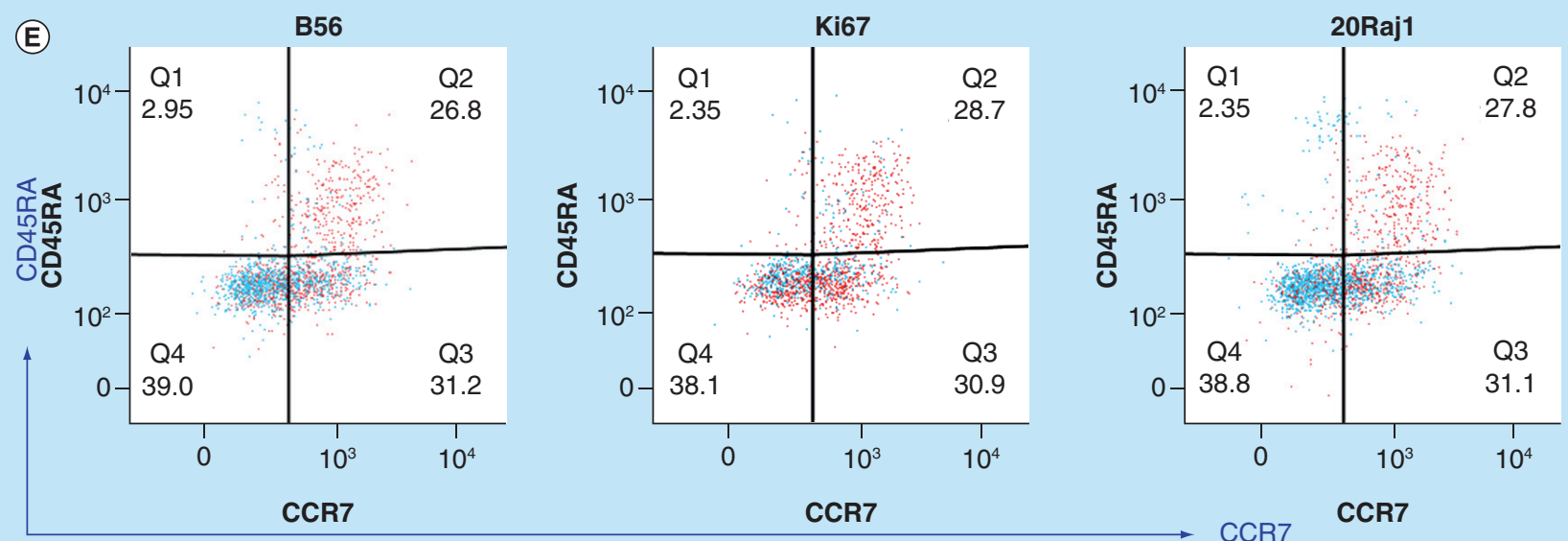

Figure 3. Comparison of different Ki67 clones. Four healthy donor blood specimens were stained in parallel using clone B56 or Ki67 or 20Raj1. The averages of $\mathrm{Ki} 7^{+}$percentage in $\mathrm{CD} 4^{+}$cells (A) and Tregs (B) were calculated. Dot plots from a representative sample are illustrated for the comparison in $\mathrm{Ki}^{+} \mathrm{CD}^{+}{ }^{+}$cells (C) and $\mathrm{Ki} 67^{+}$Tregs (D). (E) shows the distribution pattern of Ki67 ${ }^{+}$cells (blue dots) in $\mathrm{CD}^{+}$cells (red dots) after back gating. The experiment was repeated three-times. 
dots) were in the effector and central memory subsets, and only a few in naive and terminally differentiated effector memory CD45RA+ subsets. The Ki67 clonedependent difference in the frequency of $\mathrm{Ki} 67^{+}$cells in a given cell subset is important when we compare generated Ki67 data across studies and interlab studies. The Ki67 clone from BioLegend is the prototype monoclonal antibody that discovered Ki67. It has been used in other applications such as immunohistochemistry and western blot $[21,22]$. Clone B56 [13-15,17] and 20Raj1 [16] have been mostly applied for flow cytometry assays.

\section{Comparison between the Cyto-Chex ${ }^{\circledast}$ BCT \& sodium heparin tube}

Extended sample stability of blood specimens that allows transportation of blood samples to a clinical flow cytometry laboratory, is critical to incorporate markers of interest in global clinical studies. There are several publications reporting that the Cyto-Chex BCT blood collection tube has the ability to extend blood specimen stability up to 7 days after collection for many common immune phenotypic markers such as CD3, CD4, CD8, CD16/56, CD19, CD45, CD45RA, CD45RO, CD25, etc $[19,23]$. To verify each marker's phenotypic integrity in the Cyto-Chex BCT, it is necessary to compare with other common blood collection tubes such as sodium heparin during any particular assay development and validation [18]. We assayed blood samples collected in the Cyto-Chex BCT and sodium heparin tube in parallel within 2 $\mathrm{h}$ postcollection. The percentage differences in the reportable results between the blood collection tubes were within $20 \%$, as shown in Figure 4A. Some rare populations tended to have larger differences. The percentage difference in frequency of $\mathrm{Ki}^{+} 7^{+}$in $\mathrm{CD} 4^{+}$,

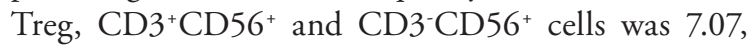
10.6, 17.4 and 3.4\%, respectively. Representative dot plots of $\mathrm{Ki}^{2} 7^{+}$cells in $\mathrm{CD}^{+}$and Treg cells are shown in Figure $4 \mathrm{~B}$ for the Cyto-Chex BCT, and Figure $4 \mathrm{C}$ for the sodium heparin tube. Based on the above results, the Cyto-Chex BCT was chosen for the assay development and clinical validation, which greatly increased the probability of analyzing blood samples within the established stability.

\section{Sample stability}

We evaluated sample stability by calculating percentage changes between the stored specimen value at given time points after collection and the fresh specimen value that was processed within $2 \mathrm{~h}$ after collection. Four healthy donor blood samples collected in the Cyto-Chex BCT were evaluated, up to $96 \mathrm{~h}$ after collection. Within $72 \mathrm{~h}$ after collection, the percentage changes in frequencies of subpopulations of interest were within $20 \%$, as compared with the fresh sample (Figure 5A, B \& C). For samples that were processed $96 \mathrm{~h}$ after collection, the percentage changes became $>20 \%$ for some of the subsets (Figure 5D). Rare populations demonstrated variable stability, probably due to the imprecision of detecting these low-frequency populations. When low numbers of cells are present in populations, a slight change in cell number can result in a large percentage difference. Processing of clinical samples cannot occur until 1 day postcollection as a result of shipping logistics of specimens. A 72-h cut-off of the sample stability was determined for this assay [18]. The dot plots from a representative sample are shown in Figure $5 \mathrm{E}$ for $\mathrm{CD} 25^{\text {high }} \mathrm{CD} 127^{\text {low }}$ Tregs from $\mathrm{CD} 4^{+}$ cells, and $5 \mathrm{~F}$ for $\mathrm{Ki} 67^{+}$Treg cells.

\section{Intra-assay \& interassay precisions}

We assessed intra-assay precision by measuring blood samples collected in the Cyto-Chex BCT. Four healthy donor blood samples were run in triplicates. The CV value was calculated for each subpopulation, and graphed in Figure 6A. All the CV values were within $10 \%$, except for the $\mathrm{Ki} 67^{+} \mathrm{CD} 4^{+}$cells, for which the CV was $10.9 \%$, due to low frequency of the population. A flow cytometry control lyophilized Veri-Cells PBMC was used to assess interassay precision by assaying in triplicate for each run. Five analytical runs were performed on five separate days. The mean from the triplicate was calculated for each run. The grand mean percentage $\mathrm{CV}$ was calculated across the five runs by dividing the grand SD by the grand mean, and graphed in Figure $6 \mathrm{~B}$ for each reportable result. An acceptance criterion of $\leq 25 \% \mathrm{CV}$ was met for all reportable results [11]. Thus, the assay had acceptable intra-assay and interassay precisions. As lyophilized Veri-Cells PBMC control has an extended stability, it can be used as a quality control to monitor daily assay performance.

\section{Interanalyst variability}

To assess interanalyst variability, two qualified individuals processed blood specimens from four healthy donors in parallel. The percentage differences between them were within $20 \%$ for all the subpopulations of interest, as shown in Figure $6 \mathrm{C}$. The percentage differences tended to be higher for those subpopulations that are present at lower frequencies.

\section{Prepared sample stability}

We compared the data acquired upon processing and storage for 4- and 24-h postpreparation. Figure 7A shows the comparison data obtained from stained samples between 0 - and 4-h postpreparation. 
(A)

Cytochex vs sodium heparin tube

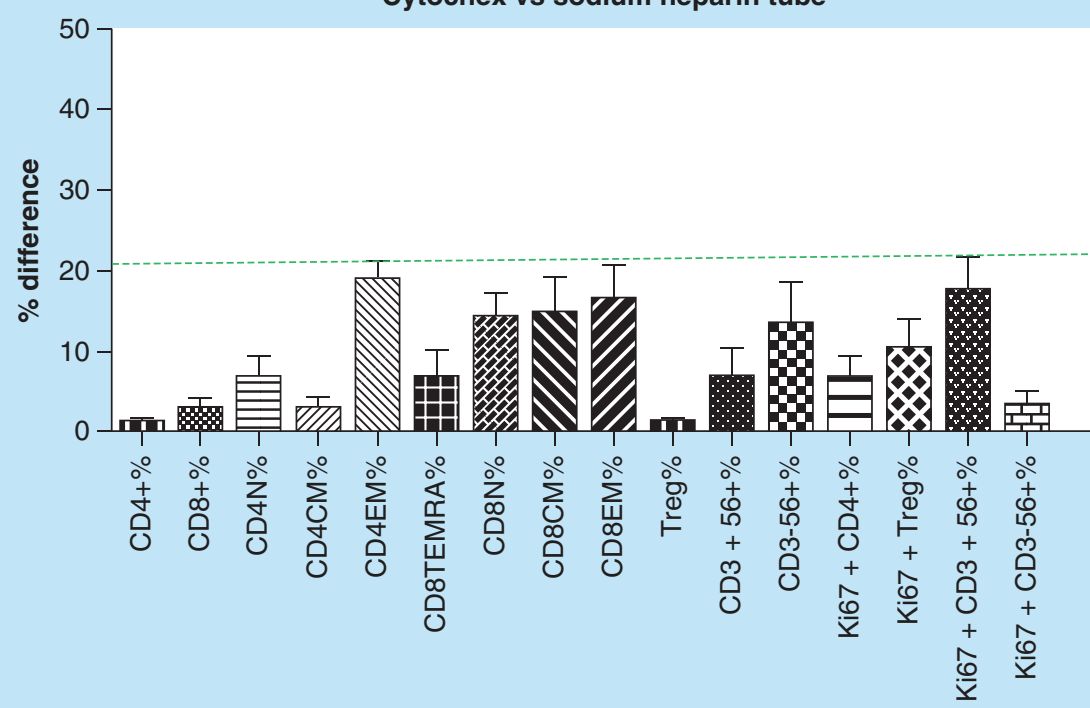

(B)

CD4+

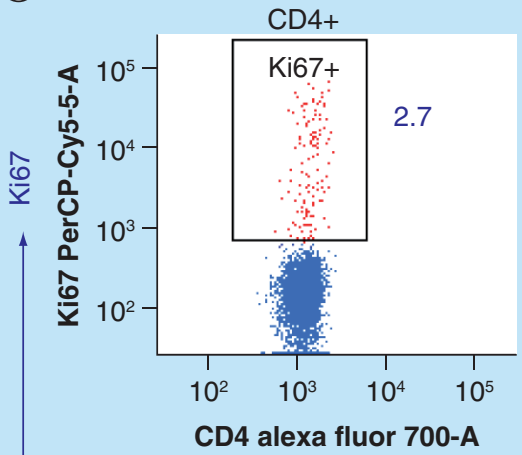

Treg

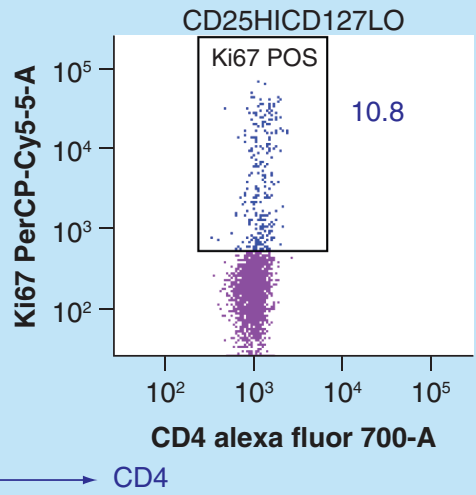

(C)

CD4+

Treg
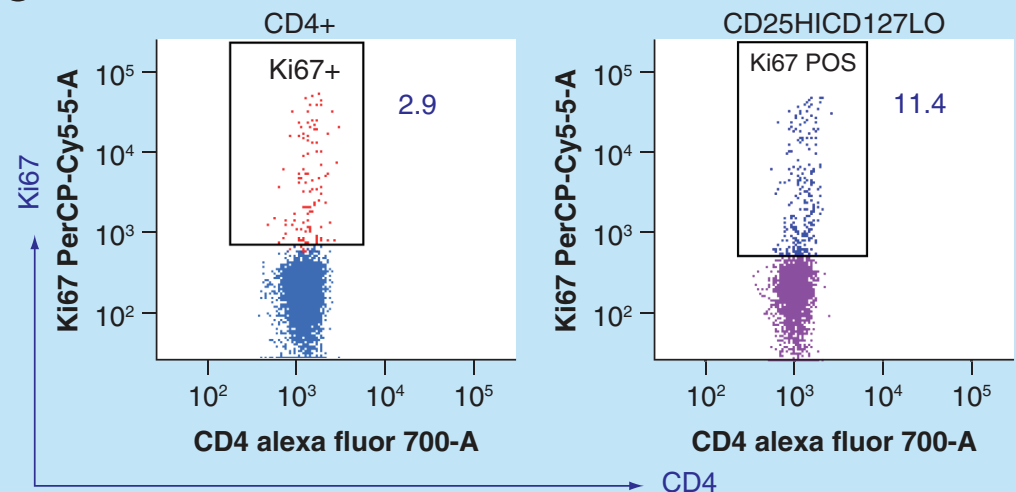

Figure 4. Comparison between the Cyto-Chex ${ }^{\circledR}$ BCT and sodium heparin tube. Four healthy donor blood specimens collected in either Cyto-Chex BCT or sodium heparin tubes were processed in parallel, and acquired using the same instrument settings. Averages of percentage differences for the subpopulations of interest were calculated and graphed (A). The green dot line indicates the $20 \%$ cut-off. Dot plots of $\mathrm{Ki}^{6} 7^{+}$cells in $\mathrm{CD} 4{ }^{+}$cells and Tregs from a representative blood sample collected in the Cyto-Chex BCT (B) and sodium heparin tube (C) are shown. The experiment was repeated twice. Numbers in the dot plots denote frequencies of Ki67+ cells in either CD4 ${ }^{+}$or Treg cells. 
The percentage changes for most subpopulations were within $10 \%$. Higher percentage changes were observed in rare populations such as $\mathrm{CD}^{+} \mathrm{CD} 56^{+}(13.5 \%)$ and $\mathrm{Ki}^{2} 7^{+} \mathrm{CD} 3{ }^{+} \mathrm{CD} 6^{+}(11.4 \%)$ cells. An acceptance criterion of $20 \%$ was met for the reportable results. If the prepared samples were acquired $24 \mathrm{~h}$ poststaining, all the markers except Ki67 were stable. The frequencies of $\mathrm{Ki} 67^{+}$in the subsets of interest were $30.6-42.4 \%$ lower than those from the samples acquired upon processing (Figure 7B). Therefore, the stained samples should be acquired within $4 \mathrm{~h}$ after preparation.

\section{Conclusion}

These experiments demonstrate that a fit-for-purpose flow cytometry assay to measure intracellular Ki67 expression in various immune cell subsets from human blood has been developed, and performs with excellent intra-assay, interassay and interanalyst precisions. Specimens can be received and analyzed up to $72 \mathrm{~h}$ when collected in the Cyto-Chex BCT tube and shipped/stored at ambient temperature. Prepared samples should be acquired within $4 \mathrm{~h}$ after processing. The assay has proven to be quantitative, reproducible and sufficiently robust to analyze samples from global clinical studies. Easy access to peripheral blood enables continuous measurement of Ki67 expression in blood as a biomarker. The assay will be applied to higher order panels monitoring broader immune cell subsets, and will be useful in drug development for immuno-oncology or immunological diseases.

\section{Future perspective}

Techniques and technologies for the analysis of large numbers of individual cells are rapidly developing;
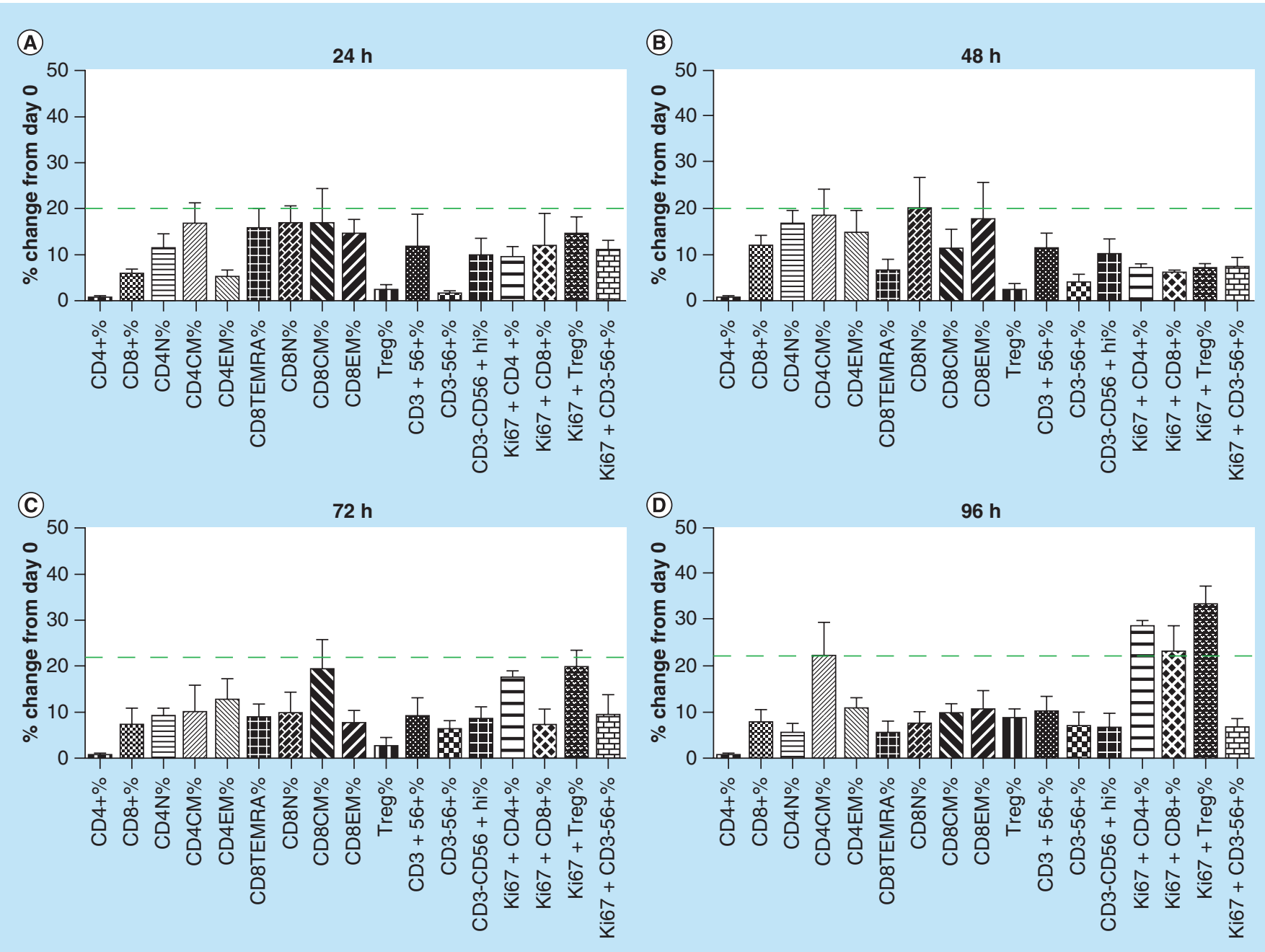

Figure 5. Assessment of sample stability. Four healthy donor blood specimens stored in the Cyto-Chex ${ }^{\circledR}$ BCT were processed at different time points after collection. Percentage changes from the fresh sample for the subpopulations of interest were calculated, and the averages of percentage changes from four healthy donors were graphed for time points of 24 (A), 48 (B), 72 (C) and $96 \mathrm{~h}$ (D). The green dot line indicates the $20 \%$ cut-off. (Facing page) Dot plots of Tregs (E) and Ki67+ Tregs (F) were from a representative sample assayed at fresh (within $2 \mathrm{~h}$ ), 24, 48 and $72 \mathrm{~h}$ after blood collection. The experiment was repeated three-times. 


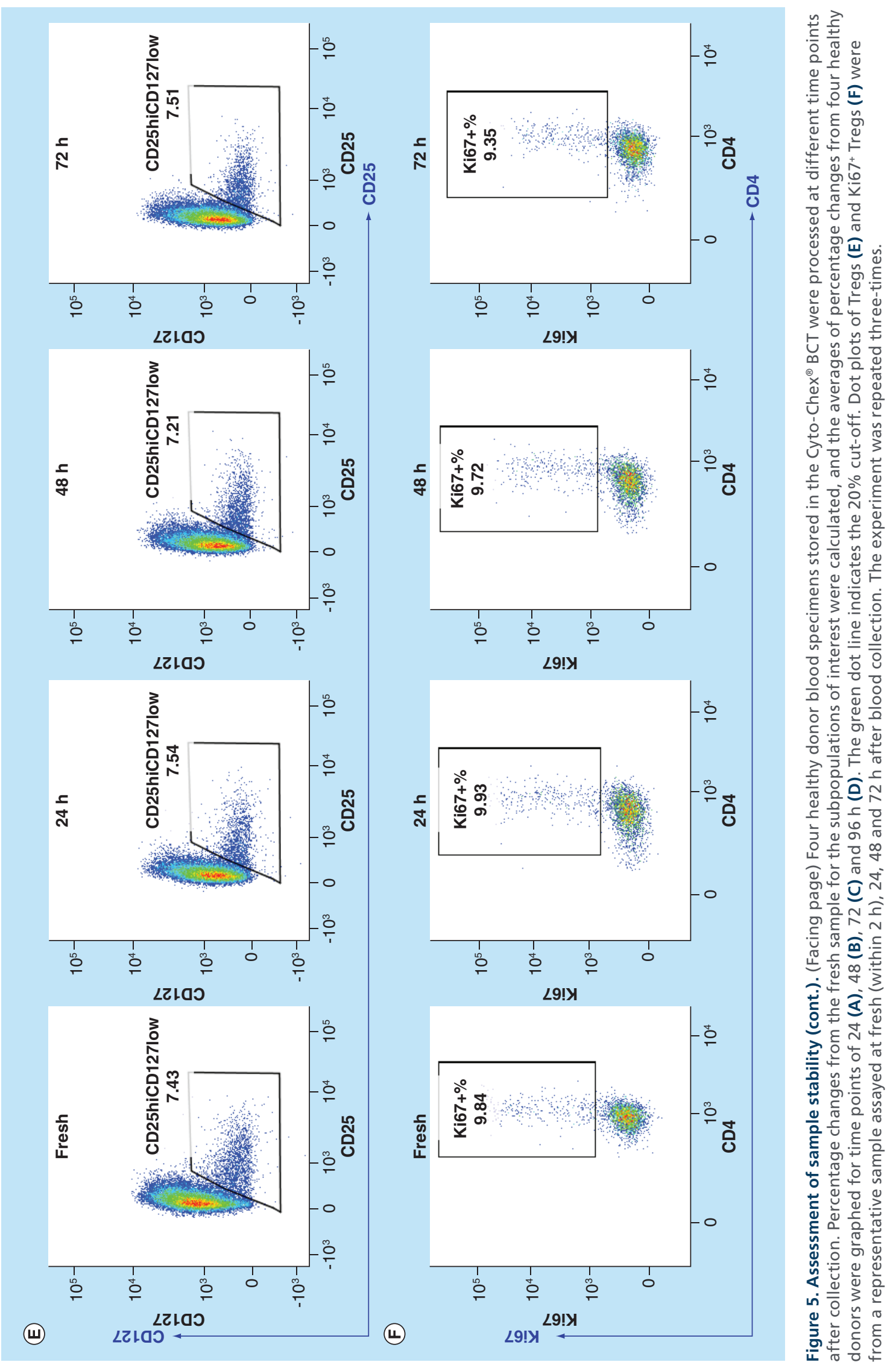




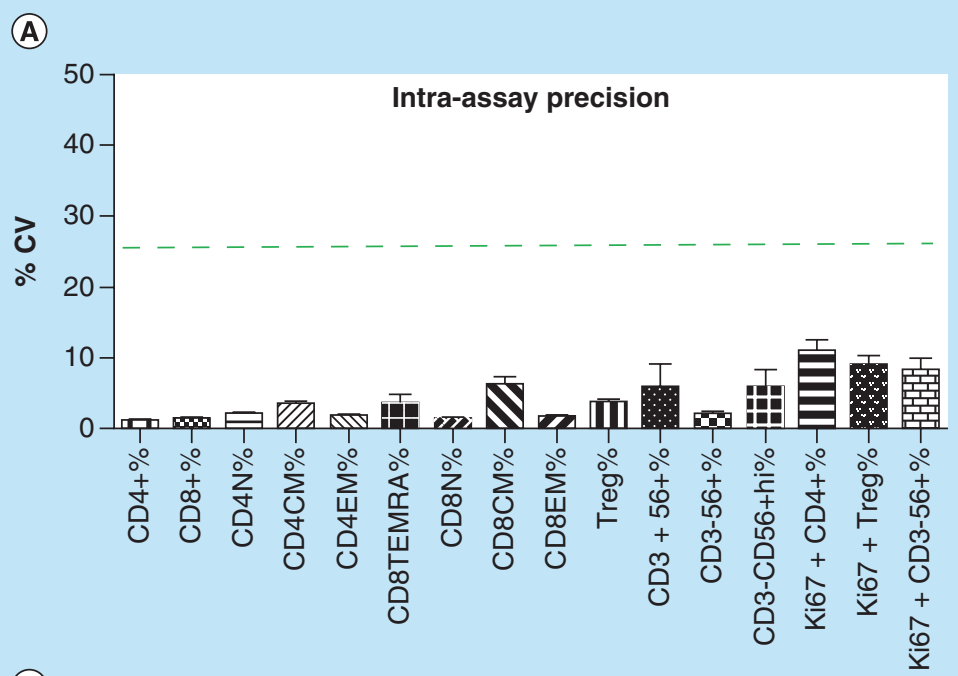

(B)

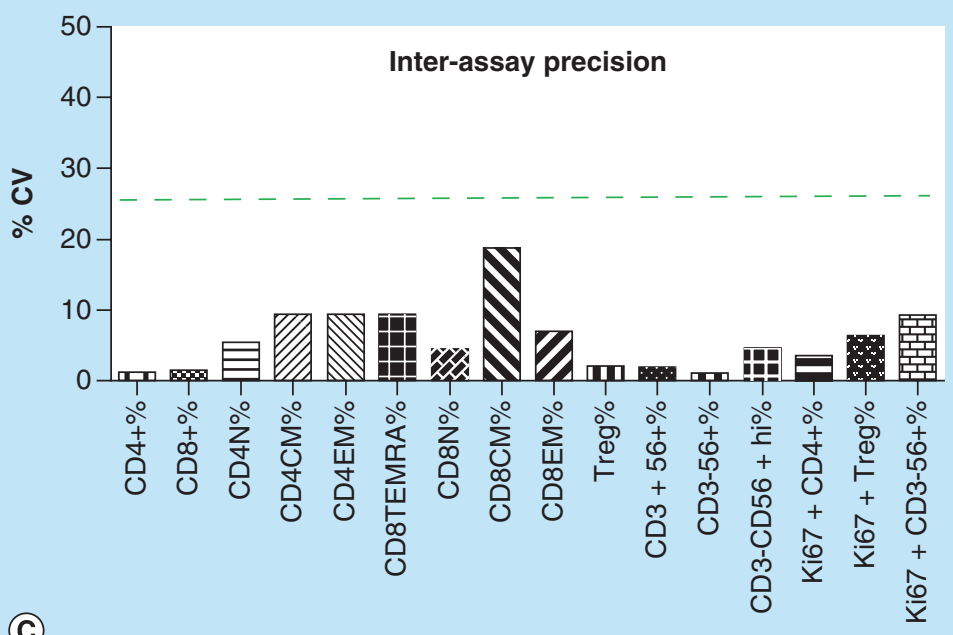

(C)

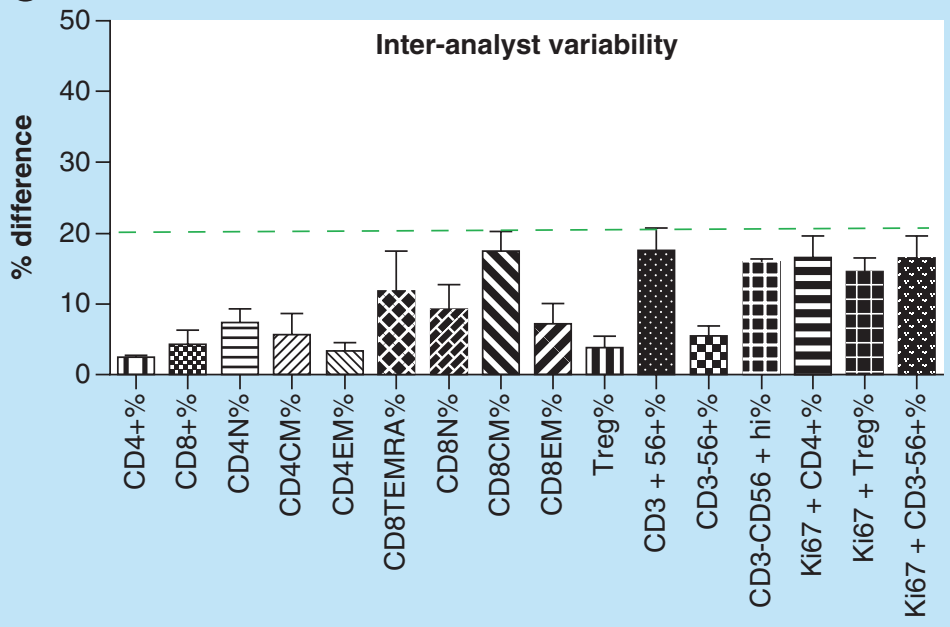

Figure 6. Assay precisions. (A) Intra-assay precision. The assay was performed in triplicates. The percentage CV was calculated for each subset across the triplicates. Averages of percentage CVs from four healthy donor blood specimens were calculated and graphed. The experiment was repeated twice. (B) Inter-assay precision. Lyophilized Veri-CellTM PBMC was assayed in triplicate for each run for a total of five analytical runs on five separate days. The mean was calculated from the triplicate for each run. The grand mean percentage CV across the five runs was calculated by dividing the grand SD by the grand mean, and graphed for each reportable result. The green dot line indicates the $25 \%$ cut-off. (C). Interanalyst variability. Four healthy donor blood specimens were assayed in parallel by two trained individuals. Averages of percentage differences between them were calculated and graphed. The green dot line indicates the $20 \%$ cut-off. The experiment was repeated twice. SD: Standard deviation. 
and flow cytometry is a prominent technology of this kind. This is largely owed to its being a key tool for understanding the immunome - an understanding required, for example, in the race to maximize the impact of immuno-oncology on the survival of cancer patients. For clinical assays, the major shortcoming of flow cytometry has always been that it is difficult to reproduce, and the stability of typical flow cytometry samples (mainly blood) is very short. However, flow cytometry innovations are being implemented in the clinic at an unprecedented speed. The design and improvement of tools to move flow cytometry methods from an individual art to a true mineable '-omics' are visible in every building block of a full-flow cytometry experiment. For example, new aggressively multiplexing instruments, fluorophores, sample stabilization methods, modalities, software, etc., are coming together to allow this powerful, yet seemingly unscalable method to become mainstream.

Individual method improvements, such as the Ki67integrating panel described here are an important step toward flow cytometry assays, which can be fully reproduced, independent of instruments or circumstances. This, in turn, is the decisive change allowing the creation of broadly used simplified point-of-care methods. Data from such methods will become the basis of the flow cytometry databases of the future. Since 2011, the American Association of Pharmaceutical Scientists Flow Cytometry Action Program Committee has published recommendation papers with the hope that these will be
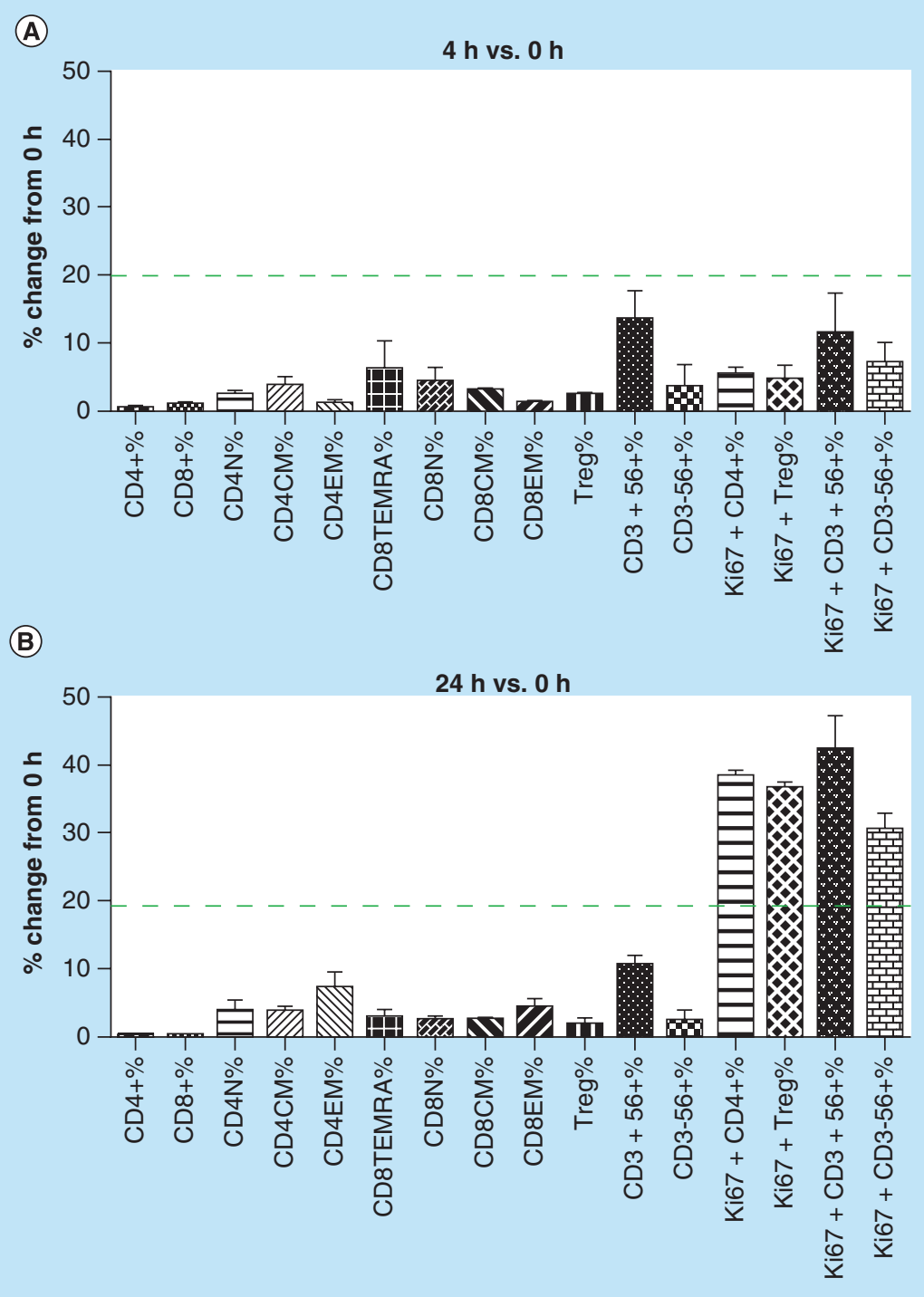

Figure 7. Prepared sample stability. Stained samples were acquired upon processing $(0 \mathrm{~h})$ and storage at $4^{\circ} \mathrm{C}$ for 4 and $24 \mathrm{~h}$ after preparation. Percentage changes from $0 \mathrm{~h}$ were calculated for the subpopulations of interest, and the averages from four healthy donors were graphed for $4 \mathrm{~h}$ (A) and $24 \mathrm{~h}$ (B). The green dot line indicates the $20 \%$ cut-off. The experiment was repeated twice. 
incorporated as official guidance documents for the validation of flow cytometric methods. Some key recommendation papers were cited in this manuscript $[11,18]$. However, unlike ligand-binding assays and mass spectrometry, there are still no official guidance documents [24]. The mission of the American Association of Pharmaceutical Scientists flow cytometry Action Program Committee is in alignment with that of the International Clinical Cytometry Society and the European Society for Clinical Cell Analysis [25]. Demonstrating the steps taken to successful implementation of robust clinical flow cytometry assays as in this example will support this effort toward a generally shared standard.

Specifically, with Ki67 being an established marker for cell proliferation in tumor tissues, the stable validated clinical assay described here for detection of Ki67 in blood, is becoming an important tool in investigating the relationship between changes in peripheral/circulating cell populations and cells in the tumor microenvironment. The Ki67 flow cytometry assay presented here has already been implemented in clinical studies of several Bristol-Myers Squibb immuno-oncology targets in cancer patients for exploratory biomarker studies. The generated biomarker data are planned to be published in the future. If a clear reproducible relationship between the tumor microenvironment and a peripheral cell population can be established, this assay will deliver a powerful biomarker in blood, an easily accessible tissue.
Acknowledgements

The authors thank L Patti-Diaz at Bristol-Myers Squibb for providing blood samples, and M Hedrick at Bristol-Myers Squibb for thoughtful discussions.

\section{Open access}

This work is licensed under the Creative Commons Attribution-NonCommercial 4.0 Unported License. To view a copy of this license, visit http://creativecommons.org/licenses/bync-nd/4.0/

Financial \& competing interest's disclosure

All authors are either employees and/or shareholders of Bristol-Myers Squibb, Co. or Aerotek Scientific Resources. The authors have no other relevant affiliations or financial involvement with any organization or entity with a financial interest in or financial conflict with the subject matter or materials discussed in the manuscript apart from those disclosed.

No writing assistance was utilized in the production of this manuscript.

\section{Ethical conduct of research}

The authors state that they have obtained appropriate institutional review board approval or have followed the principles outlined in the Declaration of Helsinki for all human or animal experimental investigations. In addition, for investigations involving human subjects, informed consent has been obtained from the participants involved.

\section{Executive summary}

Aim

- A robust Ki67 flow cytometry assay is needed to support global clinical trials.

Results \& discussion

- A flow cytometry assay to measure intracellular Ki67 expression in various immune cell subsets from human blood has been developed with optimal assay conditions.

- The assay can consistently detect Ki67 expression in various immune cell subsets tested during validation.

- The assay has been validated with excellent precisions and sample stability up to 72-h postcollection, indicating it is sufficiently robust to analyze samples from global clinical studies.

\section{Conclusion}

- The developed and validated assay will be useful in development of drugs that target immune cells to treat cancers and autoimmune diseases.

\section{References}

Papers of special note have been highlighted as:

- of interest; $\bullet$ of considerable interest

1 Topalian SL, Weiner GJ, Pardoll DM. Cancer immunotherapy comes of age. J. Clin. Oncol. 29(36), 4828-4836 (2011).

2 Topalian SL, Taube JM, Anders RA, Pardoll DM. Mechanism-driven biomarkers to guide immune checkpoint blockade in cancer therapy. Nat. Rev. Cancer 16, 275-287 (2016).

3 Hodi FS, O'Day SJ, McDermott DF et al. Improved survival with ipilimumab in patients with metastatic melanoma. $N$.
Engl. J. Med. 363(8), 711-723 (2010).

4 Borghaei H, Paz-Ares L, Horn L et al. Nivolumab versus docetaxel in advanced nonsquamous non-small-cell lung cancer. N. Engl. J. Med. 373(17), 1627-1639 (2015).

5 Yuan J, Hegde PS, Clynes R et al. Novel technologies and emerging biomarkers for personalized cancer immunotherapy. J. Immunother. Cancer 4(3), doi:10.1186/s40425-016-0107-3 (2016) (Epub ahead of print).

- Provides a nice overview of emerging biomarkers and novel technologies including flow cytometry for cancer immunotherapy. 
6 Ma W, Gilligan BM, Yuan J, Li T. Current status and perspectives in translational biomarker research for PD-1/ PD-L1 immune checkpoint blockade therapy. J. Hematol. Oncol. 9(1), 47 (2016).

7 Scholzen T, Gerdes J. The Ki-67 protein: from the known and the unknown. J. Cell. Physiol. 182(3), 311-322 (2000).

8 Yerushalmi R, Woods R, Ravdin PM et al. Ki67 in breast cancer: prognostic and predictive potential. Lancet Oncol. 11(2), 174-183 (2010).

9 Denkert C, Budczies J, von Minckwitz G et al. Strategies for developing Ki67 as a useful biomarker in breast cancer. Breast 24 (Suppl. 2), S67-S72 (2015).

10 Inwald EC, Klinkhammer-Schalke M, Hofstädter F et al. Ki67 is a prognostic parameter in breast cancer patients: results of a large population-based cohort of a cancer registry. Breast Cancer Res. Treat. 139(2), 539-552 (2013).

11 O'Hara DM, Xu Y, Liang Z et al. Recommendations for the validation of flow cytometric testing during drug development: II assays. J. Immunol. Methods 363(2), 120-134 (2011).

-. Reports recommendations for the validation of flow cytometry assays.

12 Litwin V, O'Gorman MR. Flow cytometry-based biomarkers in translational medicine and drug development. J. Immunol. Methods 363(2), 101-102 (2011).

13 Ehx G, Hannon M, Beguin Y et al. Validation of a multicolor staining to monitor phosphoSTAT5 levels in regulatory T-cell subsets. Oncotarget 6(41), 43255-43266 (2015).

14 Sanvito L, Tomita A, Chihara N et al. Increase of Ki- $67^{+}$ natural killer cells in multiple sclerosis patients treated with interferon- $\beta$ and interferon- $\beta$ combined with low-dose oral steroids. J. Neuroimmunol. 236(1-2), 111-117 (2011).

15 Vukmanovic-Stejic M, Agius E, Booth N et al. The kinetics of $\mathrm{CD}^{+}{ }^{+} \mathrm{Foxp}^{+}{ }^{+} \mathrm{T}$ cell accumulation during a human cutaneous antigen-specific memory response in vivo. J. Clin. Invest. 118(11), 3639-3650 (2008).

16 Jiao Y, Hua W, Zhang T et al. Characteristics of CD8 ${ }^{+} \mathrm{T}$ cell subsets in Chinese patients with chronic HIV infection during initial ART. AIDS Res. Ther. 8, 15 (2011).
17 Curti BD, Kovacsovics-Bankowski M, Morris N et al. OX40 is a potent immune-stimulating target in late-stage cancer patients. Cancer Res. 73(24), 7189-7198 (2013).

- Reports application of Ki67 flow cytometry assay in Phase I clinical trial.

18 Brown L, Green CL, Jones N et al. Recommendations for the evaluation of specimen stability for flow cytometric testing during drug development. J. Immunol. Methods 418, 1-8 (2015).

-• Reports recommendations for assessing specimen stability for flow cytometry assays.

19 Davis C, Wu X, Li W et al. Stability of immunophenotypic markers in fixed peripheral blood for extended analysis using flow cytometry. J. Immunol. Methods 363(2), 158-165 (2011).

20 Bell CJ, Sun Y, Nowak UM et al. Sustained in vivo signaling by long-lived IL-2 induces prolonged increases of regulatory T cells. J. Autoimmun. 56, 66-80 (2015).

21 Gerdes J, Lemke H, Baisch $\mathrm{H}$ et al. Cell cycle analysis of a cell proliferation-associated human nuclear antigen defined by the monoclonal antibody Ki-67. J. Immunol. 133(4), 1710-1715 (1984).

22 Schlüter C1, Duchrow M, Wohlenberg C et al. The cell proliferation-associated antigen of antibody Ki-67: a very large, ubiquitous nuclear protein with numerous repeated elements, representing a new kind of cell cycle-maintaining proteins. J. Cell. Biol. 123(3), 513-522 (1993).

23 Warrino DE, DeGennaro LJ, Hanson M et al. Stabilization of white blood cells and immunologic markers for extended analysis using flow cytometry. J. Immunol. Methods. 305(2), 107-119 (2005).

24 Du L, Grover A, Ramanan S, Litwin V. The evolution of guidelines for the validation of flow cytometric methods. Int J. Lab. Hematol. 37(Suppl. 1), 3-10 (2015).

25 Litwin V, Green C, Stewart JJ. Receptor occupancy by flow cytometry. Cytometry B Clin. Cytom. 90(2), 108-109 (2016). 
